\title{
Influencia de la presión atmosférica y temperatura ambiental en el subsuelo de la microcuenca San Ildefonso Trujillo, Perú
}

\author{
Influence of atmospheric pressure and ambient temperature on \\ the sub-floor of the San Ildefonso micro-basin Trujillo, Peru
}

\author{
Carlos Eduardo Quiroz Moreno ${ }^{1, *}$; José Mostacero -Leon ${ }^{2}$
}

\author{
1 Gerencia de Defensa Nacional, Gobierno Regional La Libertad-Av. España № 1800, Trujillo, Perú. \\ 2 Facultad de Ciencias Biológicas, Universidad Nacional de Trujillo, Perú. \\ *Autor corresponsal: cquiroz674@gmail.com (C.E. Quiroz). \\ ID ORCID de los autores \\ J. Mostacero-Leon: iD https://orcid.org/0000-0003-2556-3013
}

\section{RESUMEN}

En marzo del 2017 la Quebrada San Ildefonso, Trujillo, Perú, se activó como consecuencia de las precipitaciones pluviales generadas por el evento climático denominado El Niño Costero 2017 impactando la infraestructura urbana en la trayectoria de su cauce. El presente estudio tuvo como objetivo demostrar la relación existente entre los factores ambientales: presión atmosférica y temperatura ambiental en la fluctuación del nivel freático de la quebrada San Ildefonso determinando que la variación de unos pocos hectopascales (hPa) de presión, así como el incremento de la temperatura influyen en la fluctuación del nivel freático expuesto bajo la superficie del terreno. La metodología consistió en la medición de los parámetros ambientales durante los años 2018 y nivel de la napa freática de un pozo artesiano en desuso ubicado en el cauce de la quebrada durante los meses de enero a julio 2019. Los datos fueron tomados desde la superficie del terreno hasta el nivel de agua dentro del pozo cada seis horas, acorde a la oscilación máxima y mínima de la marea barométrica; simultáneamente se registraron la temperatura de aire, del agua y suelo. Se concluye que la presión atmosférica y temperatura influyen en el ascenso y descenso diario del nivel del agua subterránea de la quebrada de San Ildefonso.

Palabras clave: Quebrada San Ildefonso; nivel freático; presión atmosférica; temperatura ambiental.

\section{ABSTRACT}

In March 2017, the Quebrada San Ildefonso, Trujillo, Peru, was activated as result of the rainfall generated by the climatic event called El Niño Costero 2017 impacting the urban infrastructure during its channel. The present study aims to demonstrate the relationship between environmental factors: atmospheric pressure and ambient temperature in the fluctuation of the water table of the San Ildefonso gorge, determining that the variation of a few hectopascals ( $\mathrm{hPa}$ ) of pressure as well as the increase in the temperature influences the fluctuation of the water table exposed below the ground surface. The methodology consisted of the measurement of the environmental parameters during the years 2018 and the level of the water table of a disused artesian well located in the channel of the ravine during the months of January to July 2019. The data were taken from the surface of the terrain to the water level inside the well every six hours according to the maximum and minimum oscillation of the barometric tide; Simultaneously, the air, water and soil temperature were recorded. It is concluded that atmospheric pressure and temperature influence the daily rise and fall of the groundwater level of the San Ildefonso.

Keywords: Quebrada San Ildefonso; water table; atmospheric pressure; environmental temperature. 


\section{INTRODUCCIÓN}

Durante episodios de intensas lluvias en la provincia de Trujillo, las escorrentías generadas por la pluviosidad sobre la microcuenca de la Quebrada de San Ildefonso descienden desde el embudo de erosión (cuenca de recepción) a través de los tributarios de primer nivel hasta el cauce de transporte. Medina et al. (2017) argumenta que el terreno existente en el cauce de la quebrada está conformado por arenas, limos y arcillas, distribuidos en forma caótica y de pobre consolidación, materiales que identificarían a esta área la presencia de acuíferos porosos (Fuentes, 2012). El nivel de agua subterránea va ascendiendo, pudiendo alcanzar la superficie del terreno si la temperatura ambiental alcanza niveles superiores a $\operatorname{los} 30^{\circ} \mathrm{C}$ con un descenso de la presión barométrica menor a $6 \mathrm{hPa}$ en pozos abiertos o grietas expuestas. Estos volúmenes de agua freática emergente se sumarian a los volúmenes de lluvia que desciende desde las vertientes de la quebrada a su cauce de transporte aumentando el caudal.

Durante el verano del año 2017 en la costa norte del litoral peruano se desarrolló un evento climático extremo que por sus características océano atmosféricas se calificó como "Niño Costero 2017" por El Estudio Nacional del Fenómeno El Niño ENFEN (2017). En el litoral marino de la provincia de Trujillo frente al balneario de Huanchaco los días del 11 al 25 de marzo del 2017 la temperatura superficial del mar (TSM) se incrementó de $22,37^{\circ} \mathrm{C}$ y anomalía térmica (AT) de $+3.53^{\circ} \mathrm{C}$ (09 de marzo) a $25,63^{\circ} \mathrm{C}$ y AT de +6.80 (11 de marzo) alcanzando la temperatura critica de $27,0^{\circ} \mathrm{C}$ y $\mathrm{AT}+8.29^{\circ} \mathrm{C}$ el día 14 de marzo, condiciones ambientales que generaron lluvias sobre la provincia de Trujillo y alrededores no menores de $25 \mathrm{~mm}$ como lo registro la Estación Meteorológica Automática (EMA) Davis del Proyecto Huacas de Moche. Los días sucesivos la temperatura superficial del mar siguió ascendiendo hasta alcanzar los $29,27^{\circ} \mathrm{C}$ y AT $+10,49{ }^{\circ} \mathrm{C}$ el 16 de marzo como lo reporta el Instituto del Mar del Perú IMARPE (2017). Este nivel térmico extremo de la TSM y su AT no se registraba en nuestro litoral nacional $y$ particularmente regional en los últimos 19 años posteriores al evento El Niño Oscilación Sur (ENOS) suscitado en febrero del año 1998. Como consecuencia de este evento climático intensas lluvias precipitan sobre la cuenca orográfica de los valles del litoral de La Libertad activando quebradas ubicadas al este de la planicie costanera de la provincia de Trujillo, así como incrementando el cauce de los ríos Moche y Chicama. El Centro Nacional de Estimación, Prevención y Reducción del Riesgo de Desastres. CENEPRED (2017) considera este evento pluvial como "Extremadamente lluvioso" superando en frecuencia e intensidad las lluvias registradas en los años "Niño 1982-83" y "Niño 1997-98". Las escorrentías y efluentes generados en el cauce de la quebrada San Ildefonso impactaron con sus sedimentos tres distritos de la provincia de Trujillo y la metrópoli misma, ocho activaciones durante los días del 15 al 22 de marzo del año 2017 Proyecta Consultoría (2018a).

Los estudios de hidrología en cuencas áridas y particularmente en nuestro litoral costanero son aun incipientes al plantearse más preguntas que respuestas en su complejo ciclo hidrológico no existiendo estudios para identificar los procesos en la distribución espacial de la lluvia, su infiltración, recarga de acuíferos y su interacción con los factores ambientales imperantes.

La presente investigación se realizó ante el limitado conocimiento en la interacción de la presión barométrica, temperatura ambiental y su influencia en el ascenso y descenso diario del nivel freático de los acuíferos subterráneos de la microcuenca de San Ildefonso. Estas variables ambientales intensifican su dinámica cuando se presentan condiciones climáticas extremas como en eventos El Niño asociado a intensa pluviosidad, favoreciendo el incremento freático y afloramiento superficial de agua subterránea hacia el cauce de la quebrada la misma que con la lluvia presente en estas condiciones aumenta sustancialmente el caudal de la quebrada. El presente estudio está orientada a demostrar la relación existente entre los factores ambientales: presión atmosférica y temperatura ambiental en la fluctuación del nivel freático en el lecho de la quebrada San Ildefonso, distrito de El Porvenir, provincia de Trujillo.

Por lo descrito anteriormente y no habiéndose realizado trabajos sobre estos factores climáticos en la zona de estudio, la presente investigación se avoco a determinar la influencia de la presión atmosférica y la temperatura ambiental en el ascenso y descenso del agua freática en el cauce de la quebrada San Ildefonso, distrito del Porvenir, provincia de Trujillo., para un mejor entendimiento de las fuerzas climáticas en la activación fluvial de las quebradas costeras.

\section{MATERIAL Y MÉTODOS}

\section{1. Área de estudio}

El presente estudio se desarrolló en las

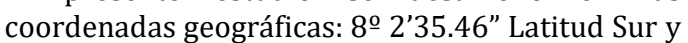
78 59' 5.96" Longitud Oeste a una altura de 192 m.s.n.m sobre el lecho de la microcuenca San Ildefonso, distrito de El Porvenir, provincia de Trujillo. Esta formación geológica está ubicada en la vertiente occidental de la cordillera de los andes, emplazada en la intercuenca 137719 de la vertiente del Pacifico; limita hacia el sur con la Cuenca Hidrográfica del Moche y por el norte de su ubicación con la Cuenca Hidrográfica del Chicama. 2.2 Técnica e instrumentos de recolección de datos

Registro mensual de temperatura ambiental, temperatura del suelo en el área de estudio.

Registro mensual de la presión barométrica en el área de estudio. 
Medición de la napa freática en el pozo artesiano ubicado en el lecho de la quebrada San Ildefonso Registros meteorológicos del Complejo arqueológico Huacas de Moche, Complejo Arqueológico Chan Chán, Servicio de Meteorología y Aeronáutica CORPAC, ONERN-Autoridad Nacional del Agua e información pluviométrica proveniente del Servicio Nacional de Meteorología e Hidrología (SENAMHI, 2014, 2106).

Medición de datos. Se tomaron mediciones del nivel freático del pozo de manera directa, esto es mediante una wincha métrica se midió la distancia entre el nivel del suelo preestablecido y la superficie del agua del pozo. Las primeras mediciones se realizaron entre las 08:50 a 11:00 horas y la segunda medición entre las 15:00 a las 16:00 horas, siguiendo la variación máxima y mínima de la marea barométrica. En cada medición se tomó la presión barométrica, la temperatura del aire, la temperatura del suelo introduciendo el bulbo del termómetro $2 \mathrm{~cm}$ bajo el terreno, la temperatura de la roca se realizó colocando el sensor del termómetro sobre la superficie de la piedra. La temperatura del agua del pozo se realizó extrayendo agua del pozo mediante una jarra plástica de 2 litros de capacidad e inmediatamente introduciendo dentro de la columna de agua el termómetro digital y procediéndose a registrar la lectura.

Análisis de datos. La información recabada fue organizada en tablas sometiendo las variables de estudio: temperatura del aire y presión barométrica a la prueba estadística conocida como "coeficiente producto - momento" Hernández et al. (2014). El coeficiente de correlación de Pearson determino el vínculo entre ellas.

$$
\begin{gathered}
r_{x y}=\frac{n \cdot \sum x_{i} \cdot y_{i}-\sum x_{i} \cdot \sum y_{i}}{\sqrt{\left[n \cdot \sum x_{i}^{2}-\left(\sum x_{i}\right)^{2}\right] \cdot\left[n \sum y_{i}^{2}-\left(\sum y_{i}\right)^{2}\right]}} \\
-1 \leq r \leq 1
\end{gathered}
$$

Dónde:

rxy: Coeficiente de correlación de Pearson; X: La variable independiente de intervalo / razón: Temperatura del aire; Y: La variable dependiente de intervalo / razón: Presión barométrica, y N: Tamaño de la muestra.

Se empleó para el procesamiento el programa Excel 2013 Análisis de datos -Funciones para Análisis Coeficiente de correlación.

\section{RESULTADOS Y DISCUSIÓN}

La quebrada de San Ildefonso es una formación geológica con un área de recepción pluvial de 10,7 $\mathrm{km}^{2}$ y con un perímetro de $18,6 \mathrm{~km}^{2}$ en forma elipsoidal ubicada a $8 \mathrm{~km}$ al noreste del continuum urbano de la ciudad de Trujillo (Apoyo Consultora, 2018).

Esta quebrada es una de las siete micro cuencas secas tributarias y no tributarias de la cuenca baja del rio Moche y que, en episodios océanoatmosféricos extremadamente cálidos como los de "El Niño" o de "El Niño Costero" cobran especial atención por los flujos que genera como resultado de las precipitaciones pluviales extraordinarias en dicho evento, efluentes pluviales que transportan solidos suspendidos en gran volumen como en El Niño 1983 donde se estimó un caudal máximo instantáneo de $26 \mathrm{~m}^{3} / \mathrm{s}$ siendo superado solo por El Niño de 1998, cuando en febrero alcanzó la quebrada San Ildefonso un máximo de $60 \mathrm{~m}^{3} / \mathrm{s}$ originando el colapso del dique de Mampuesto (Convenio UNT/INDECI, 2002).

La variación de temperatura del aire en la zona de estudio registra una tendencia inversa a los valores de la presión barométrica como se observa en la tabla y Figura 1 correspondiente a mediciones realizadas el año 2018.

Tabla 1

Registro de temperatura del aire, suelo, roca y presión atmosférica registrada en la quebrada de San Ildefonso. Periodo: enero a diciembre 2018. Altura 186 m.s.n.m. en coordenadas WGS 84: 8 02'35.46” L.S y 78 59’596" LW

\begin{tabular}{lcccccccccccc}
\hline Parámetros & Enero & Feb & Mar & Abril & May & Junio & Julio & Agos & Sept & Oct & Nov & Dic \\
\hline T Aire ${ }^{\circ} \mathrm{C}$ & 21,5 & 22,1 & 28,3 & 25,5 & 19 & 18,7 & 17,5 & 17,0 & 16,8 & 18,3 & 19,2 & 20,3 \\
T Suelo ${ }^{\circ} \mathrm{C}$ & 27,5 & 27,5 & 32,5 & 32,5 & 31,5 & 29,0 & 27,3 & 26,2 & 24,0 & 20,3 & 29,3 & 30,2 \\
T roca ${ }^{\circ} \mathrm{C}$ & 26,7 & 25,9 & 30,7 & 30,7 & 21,9 & 24,1 & 23,5 & 21,1 & 20,2 & 19,5 & 24,3 & 28,5 \\
Presión Atmos.(hPa) & 989,5 & 988,0 & 985,4 & 986,0 & 986,8 & 987,0 & 990,4 & 992,7 & 993,5 & 992,0 & 991,0 & 990,0 \\
\hline
\end{tabular}

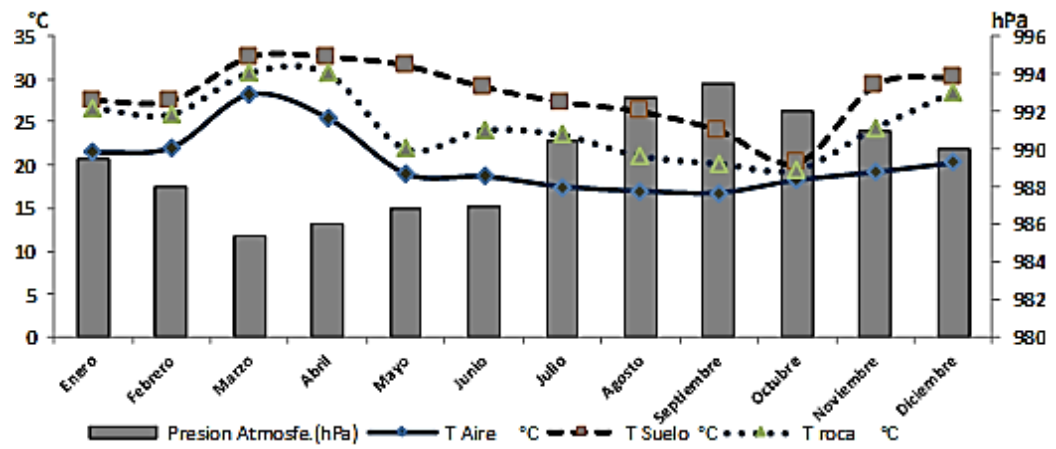

Figura 1. Comparativo de los valores de temperatura del aire, temperatura del suelo, temperatura de las rocas y presión barométrica tomados en la quebrada San Ildefonso a una altura de $192 \mathrm{msnm}$ durante los meses de enero a diciembre del año 2018. 


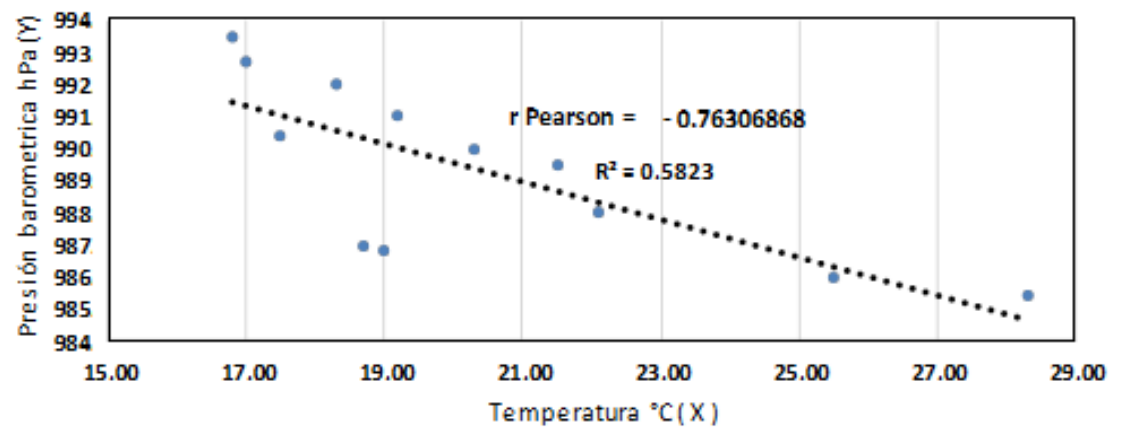

Figura 2. Relación de las variables del estudio y su tendencia

Se evidencio que los valores de ascenso y descenso del nivel freático en el lecho de la quebrada oscilan en la mañana y tarde entre los 3,90 m (31 de enero 2019) hasta los 0,20 m (18 julio 2019), concordante con la temperatura ambiental que registra sus más altos niveles en verano contrario a los valores de la presión barométrica que descienden significativamente (Tabla 3) para valores correspondientes al año 2019.

Tabla 2

Temperatura del aire $(\mathrm{X})$ y presión barométrica (y) tomada en la quebrada San Ildefonso (año 2018)

\begin{tabular}{ccc}
\hline Meses & Temp. Aire ${ }^{\circ} \mathrm{C}(\mathrm{X})$ & Presión barom. $\mathrm{hPa}(\mathrm{y})$ \\
\hline 1 & 21,50 & 989,50 \\
2 & 22,10 & 988,00 \\
3 & 28,30 & 985,40 \\
4 & 25,50 & 986,00 \\
5 & 19,00 & 986,80 \\
6 & 18,70 & 987,00 \\
7 & 17,50 & 990,40 \\
8 & 17,00 & 992,70 \\
9 & 16,80 & 993,50 \\
10 & 18,30 & 992,00 \\
11 & 19,20 & 991,00 \\
12 & 20,30 & 990,00 \\
\hline
\end{tabular}

La relación de la presión atmosférica y el nivel de la napa freática ha sido estudiada por Raffo (1954) en Argentina, aseverando que cuando aumenta la presión atmosférica, el nivel de agua de los pozos disminuye y viceversa. Lo antes indicado ha sido corroborado en el presente estudio como se observa en la Tabla 3 y Figura 3 y 4, destacando las mayores fluctuaciones de la napa freática del pozo en el verano: El día 31 de enero 2019 a las 11:00 am en el área de estudio se registró una presión atmosférica de 991 hPa la más elevada en el día prevista en la curva sigmoidea de la marea barométrica; la temperatura ambiental fue de $33,0{ }^{\circ} \mathrm{C}$ y la temperatura del suelo de $38,9^{\circ} \mathrm{C}$; bajo esas condiciones el nivel freático registro la profundidad más extrema reportada a la fecha: $9,0 \mathrm{~m}$ bajo la superficie del terreno. En la medición realizada a las 04:50 p.m. hora del punto barométrico más bajo: $989,9 \mathrm{hPa}$, con una temperatura del suelo en $29,1^{\circ} \mathrm{C}$ la medición de la napa freático registro un ascenso de 3,90 m de la columna de agua al haber alcanzado la napa freática los $5,10 \mathrm{~m}$ bajo el terreno, ello tan solo con una diferencia de $1,1 \mathrm{hPa}$ en aproximadamente 6 horas de diferencia. El 13 de marzo del 2019 a las 10:00 a.m. se reportó el máximo valor barométrico del día: $990 \mathrm{hPa}$ midiéndose la profundidad del nivel freático en 6,50 m bajo la superficie; a las 03: 15 p.m. La mínima cota barométrica alcanzo los $987,0 \mathrm{hPa}$ registrándose una medición desde el borde del terreno hasta el nivel del líquido del pozo una profundidad de $3,50 \mathrm{~m}$, esto es, entre la medición de la mañana y a la vespertina existía una diferencia de $3 \mathrm{~m}$ de la columna de agua tan solo por una variación de $3 \mathrm{hPa}$ y en un tiempo aproximado de 5:15 horas. El día 14 de marzo la quebrada de San Ildefonso registro desde la 1:00 am una intensa lluvia que alcanzó los $8,59 \mathrm{~mm}$, repitiéndose a las 2:00 y 4:00 a.m. con menor intensidad: $1,6 \mathrm{~mm}$ y $0,8 \mathrm{~mm}$ respectivamente, registrando un volumen total día de $10,99 \mathrm{~mm}$ como se observa en la Figura 5. Al evaluar los niveles y valores ambientales a las 9:00 am la presión barométrica reporto: 990,0 hPa, la temperatura del aire $36,5{ }^{\circ} \mathrm{C}$, la temperatura del agua $30,0^{\circ} \mathrm{C}$ y la del suelo $36,5^{\circ} \mathrm{C}$, el nivel freático había ascendido hasta alcanzar $0.30 \mathrm{~m}$ bajo la superficie del terreno. Al evaluar a las 04:15 p.m se registró una presión barométrica de $984 \mathrm{hPa}$ (diferencia de $6 \mathrm{hPa}$ con respecto a la primera medición del día) el nivel freático había ascendido hasta alcanzar los 0,10 m bajo la superficie del terreno como se evidencia en la Tabla 3. El sustantivo incremento del nivel freático registrado el día 14 de marzo 2019 estaría relacionado a la lluvia de $11 \mathrm{~mm}$ en las primeras horas del día y que como asevera Fuentes (2012): “Las aguas superficiales y las aguas subterráneas están muy relacionadas, pues es frecuente que el agua subterránea aflore en fuentes y manantiales para seguir un recorrido superficial, mientras que en otros casos el agua superficial se infiltra pasando a formar parte del agua subterránea (...) Las Aguas superficiales se infiltran en el terreno por los poros y grietas del suelo hasta llegar a la profundidad donde todos los huecos están llenos de agua, formando una franja capilar de mayor o menor espesor esta zona es la denominada zona de saturación o capa freática, su límite superior se llama superficie de saturación o superficie freática. El agua de la zona de saturación asciende por capilaridad por los pequeños intersticios del terreno según su naturaleza puede elevarse uno, dos o tres metros por encima de la superficie de saturación". La información procesada evidencia que durante los meses de enero a marzo (verano) la presión barométrica en el área de estudio tuvo un promedio de fluctuación de $2.4 \mathrm{hPa}$; sin 
embargo, el nivel de ascenso /descenso del agua freática alcanzo la media de 2,93 m. Contrario a ello los meses de abril a julio (otoño /invierno) la variación de la presión barométrica entre la mañana y la tarde promedio los $5,6 \mathrm{hPa}$ y una fluctuación de la napa freática no mayor de 1,00 m. La información obtenida en la quebrada de San Ildefonso indicaría que cuando los valores de la temperatura ambiental se incrementan en el verano los valores de la presión atmosférica decrecen. Podemos entender que además de la lluvia que generan flujos de agua superficial que precipita sobre el relieve de la quebrada de San Ildefonso en eventos climáticos pluviales extremos como es el caso de El Niño Costero, habría que considerar el ascenso del nivel de la napa freática en volúmenes significativos por efecto de la disminución de la presión atmosférica externa. Durante el evento El Niño Costero 2017 la precipitación pluvial más intensa registrada por las Estaciones Meteorológicas Automáticas (EMA) ubicadas en la provincia Trujillo fue el día 14 de marzo 2017 con 25.0 mm (EMA Huacas de Moche ) generando dicha precipitación el día 15 de marzo el desembalse del dique construido para esas eventualidades en la quebrada de San Ildefonso; este desembalse impacto los centros urbanos: El Porvenir, Florencia de Mora, Trujillo y Víctor Larco. Cuatro días después el 19 de marzo con una precipitación de solo $6.2 \mathrm{~mm}$ registrado por la EMA Proyecto Huacas de Moche y de $3.6 \mathrm{~mm}$ por la EMA Agroindustrial Laredo, el caudal procedente de la quebrada San Ildefonso alcanzo el record pico de $39.9 \mathrm{~m}^{3} / \mathrm{s}$ Proyecta Consultoría (2018b). Consideramos que por las condiciones ambientales presentes (baja gradiente de la presión atmosférica e incremento de la temperatura) las copiosas precipitaciones pluviales en el evento climático extremo aunado a la rugosidad del terreno y la naturaleza del sedimento de la micro cuenca constituido por gravas, arena gruesa, limos y arcillas de pobre consolidación Medina et al. (2017) típico de un acuífero poroso, el agua se habría infiltrado rápidamente hasta incrementar el manto freático el que habría ascendido alcanzado el lecho del cauce de la quebrada, aumentando el volumen de escorrentía pluvial. El efecto cíclico de ascenso y descenso de la presión ambiental se denominado "marea barométrica" característica importante en la circulación de la atmosfera media y alta observándose de ella una variación diurna y semidiurna, así como en transcurso del año con el cambio de las estaciones siendo muy marcadas en el verano y en invierno. Igualmente, estas mareas atmosféricas pueden sufrir modificaciones de hasta $50 \mathrm{hPa}$ como consecuencia de los sistemas del tiempo" (Possia et al., 2015). Las fluctuaciones de la presión atmosférica diarias explicarían la ocurrencia de lluvias veraniegas sobre la provincia en las horas donde se registran los mínimos valores de esta: 04 a 05 horas y 16:00 a 17:00 horas del día. La presencia de mayor vapor de agua (nubes) sobre el terreno y colinas disminuye la densidad del aire por que el peso molecular del agua $(18,016$ $\mathrm{Kg} / \mathrm{mol}$ ), es menor que el peso molecular promedio del aire $(28,97 \mathrm{~kg} / \mathrm{mol})$, por lo tanto, ante temperaturas similares, una masa de aire más húmedo ejerce menos presión que una masa de aire más seco, favoreciendo el ascenso de los niveles de la napa freática hacia la superficie (Zúñiga y Crespo, 2015).

Tabla 3

Registro de la presión barométrica, temperatura del aire, temperatura del agua, temperatura del suelo y nivel de la napa freática en horas de la mañana (a.m.) y en horas de la tarde (p.m.) según fechas indicadas durante los meses de enero a julio 2019 en la quebrada de San Ildefonso

\begin{tabular}{|c|c|c|c|c|c|c|c|c|c|c|}
\hline Fecha & Presión & Temp & Temp. agua & $\begin{array}{c}\text { Temp. } \\
\text { Suel }\end{array}$ & $\begin{array}{l}\text { Nivel } \\
\text { napa }\end{array}$ & Presión & $\begin{array}{l}\text { Temp } \\
\text { aire }\end{array}$ & $\begin{array}{c}\text { Temp. } \\
\text { Agua }\end{array}$ & $\begin{array}{c}\text { Temp } \\
\text { suel }\end{array}$ & $\begin{array}{c}\text { Nivel } \\
\text { napa }\end{array}$ \\
\hline & a.m & a.m & a.m & a.m & a.m & p.m & p.m & p.m & p.m & p.m \\
\hline 29-ene-19 & 992,0 & 28,0 & 29,0 & 35,8 & $-6,10$ & 988,60 & 31,0 & 29,0 & 36,5 & $-3,80$ \\
\hline 31-ene-19 & 991,0 & 33,0 & 28,5 & 38,9 & $-9,00$ & 989,90 & 28,0 & 26,9 & 29,1 & $-5,10$ \\
\hline $12-f e b-19$ & 991,0 & 28,0 & 31,0 & 33,0 & $-5,10$ & 988,30 & 33,8 & 31,0 & 37,5 & $-2,60$ \\
\hline 13-mar-19 & 990,0 & 36,0 & 28,0 & 37,4 & $-6,50$ & 987,00 & 37,4 & 29,0 & 38,5 & $-3,50$ \\
\hline 14-mar-19 & 990,0 & 36,5 & 30,0 & 37,8 & $-0,30$ & 984,00 & 37,4 & 30,5 & 38,4 & $-0,10$ \\
\hline 15-abr-19 & 993,2 & 25,0 & 28,0 & 29,0 & $-4,50$ & 988,10 & 27,7 & 27,4 & 28,0 & $-3,20$ \\
\hline 16-may-19 & 993,2 & 20,0 & 27,0 & 25,0 & $-4,10$ & 989,20 & 23,0 & 26,0 & 27,0 & $-3,00$ \\
\hline 13-jun-19 & 992,5 & 23,0 & 26,0 & 26,0 & $-3,80$ & 984,00 & 24,0 & 25,0 & 26,8 & $-2,50$ \\
\hline 18-jul-19 & 992,7 & 17,5 & 28,4 & 19,3 & $-3,57$ & 988,00 & 15,4 & 24,0 & 16,6 & $-3,37$ \\
\hline
\end{tabular}

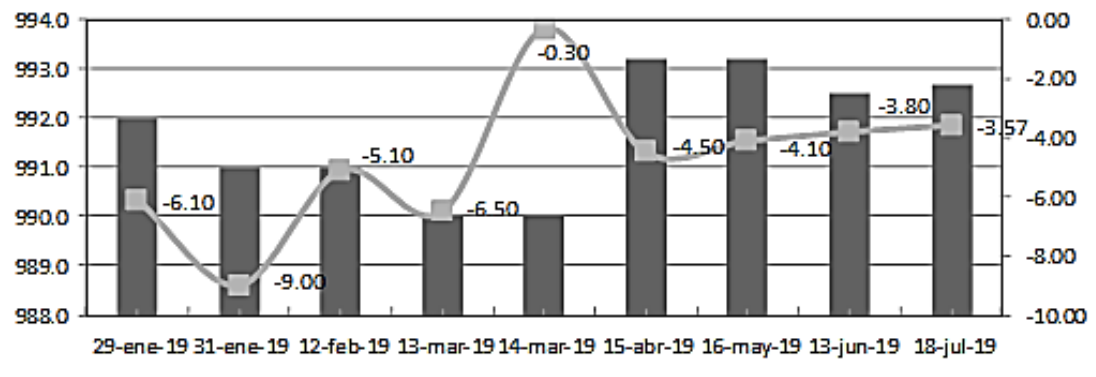

Presion barometrica (hPa) $\quad-$ - Nivel denapa freatica $(\mathrm{m})$

Figura 3. Valores de la presión barométrica y niveles de la napa freática en el área de estudio registrados en horas de la mañana (09:00 - 11:00 horas). 


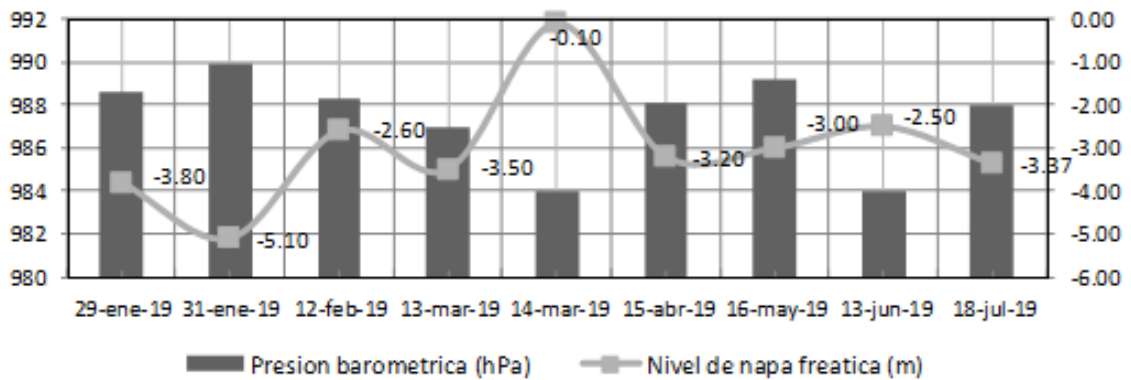

Figura 4. Valores de la presión barométrica y niveles de la napa freática en el área de estudio registrados en horas de la tarde. (15:00 - 17:00 horas).

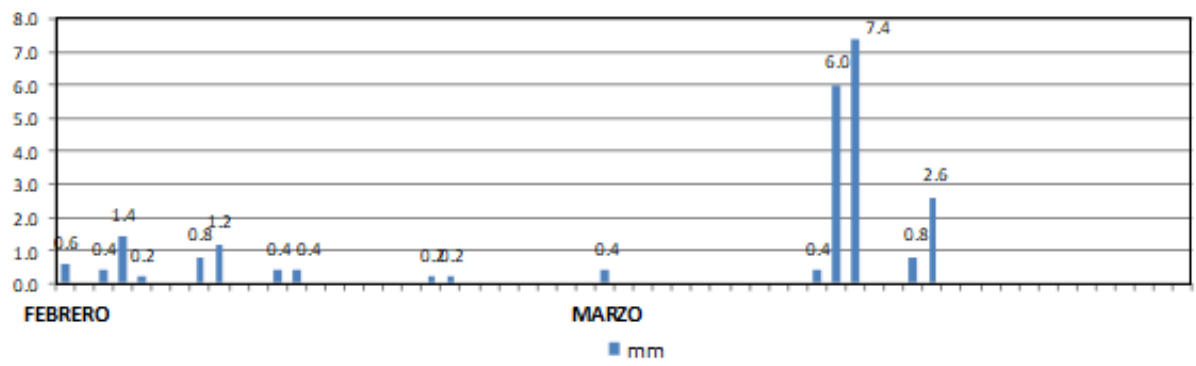

Figura 5. Niveles de lluvia registrada por la Autoridad Nacional del Agua (ANA) durante los meses de Febrero y Marzo 2019. Fuente: Estación Pluviométrica. ANA- Quebrada San Ildefonso (año 2019).

Coincidimos por lo expuesto por Ayllon (2013) “La fuerza atmosférica varía según la temperatura, la altitud y la humedad. A un aumento de temperatura corresponde una dilatación del aire y, por tanto, una disminución de presión entre las moléculas del aire y, viceversa: un descenso de temperatura produce una contracción del aire, y en consecuencia un aumento de la presión". Para establecer el grado de relación o índice de asociación lineal de las variables estudiadas: temperatura del aire $y$ presión barométrica se ha aplicado el coeficiente de correlación de Pearson a los valores obtenidos (Tabla 2 y Figura 2).

Los resultados procesados mediante la fórmula del cálculo del coeficiente de Pearson se obtuvo r: 0.763068 y un $\mathrm{R}^{2}$ de 0.5823 , lo que significa que el valor del coeficiente entre ambas es $76,3 \%$ de variación, pero en sentido inverso u opuesto considerándose dicha cifra una correlación negativa considerable (Hernández et al., 2014).

\section{CONCLUSIONES}

La temperatura del aire tiende a ser opuesta a los valores de la presión barométrica sobre el cauce de la quebrada San Ildefonso, factores que tienen influencia directa sobre la fluctuación de la napa freática.

La información procesada evidencia que durante los meses de enero a marzo (verano) la presión barométrica en el área de estudio tuvo un promedio de fluctuación de 2,4 hPa; sin embargo, el nivel de ascenso /descenso del agua freática alcanzo la media de $2.93 \mathrm{~m}$. Un episodio de lluvia de $11 \mathrm{~mm}$ suscitado el 14 de marzo 2019 evidencio el ascenso del nivel de la napa freática hasta ubicarse a $0.10 \mathrm{~m}$ del nivel del terreno. Contrario a ello los meses de abril a julio (otoño/invierno) la variación de la presión barométrica entre la mañana y la tarde promedio los $5.6 \mathrm{hPa}$ y una fluctuación de la napa freática no mayor de $1.00 \mathrm{~m}$.

Consideramos que ante el cambio climático en desarrollo y el incremento en la frecuencia pluvial sobre las quebradas costeras que ponen en vulnerabilidad los centros urbanos asentados en su cauce, se recomienda ampliar estudios que permitan evaluar las variables temperatura $\mathrm{y}$ presión barométrica y su influencia en el ascenso de los niveles freáticos y de estos en el incremento de los caudales pluviales.

\section{REFERENCIAS BIBLIOGRÁFICAS}

Apoyo Consultoría. 2018. Avance del Estudio de Pre Inversión de Proyecto "Control de Inundación en la Quebrada de San Idelfonso-Trujillo". Consorcio financista Backus, C. Pacasmayo, Minera Poderosa y Telefónica del Perú. Trujillo. 22 abril 2018

Ayllon, T. 2013. Elementos de Meteorología y Climatología. Tercera Edición. México. Editorial Trillas, 43-102 pp.

CENEPRED 2017. Informe de evaluación de Riesgos por Inundación Pluvial en el Área de Influencia El Porvenir, Provincia de Trujillo, La Libertad. Informe Técnico. Junio 2017. 20-26 pp.
ENFEN. 2017. Informe Técnico Extraordinario № 0012017/ENFEN El Niño Costero 2017. Resumen Ejecutivo. Comisión Multisectorial Encargada del Estudio Nacional del fenómeno "El Niño". Callao. Julio 2017. Perú. 1-12 pp

Fuentes, Y. 2012. Aguas subterráneas. Ministerio de Agricultura, Pesca y Alimentación, Secretaria General de Estructuras Agrarias. 2-13 pp

Hernández, R.; Fernández, R.; Baptista, P. 2014. Metodología de la Investigación. Sexta edición. McGraw Hill. México DF.300 -308 pp. 
Instituto del Mar del Perú. 2017. Boletín Diario Oceanográfico. Temperatura Superficial del Mar. Vol.4, № 076. Lima, Perú. 2$3 \mathrm{pp}$

INDECI- UNT .2002. Mapa de peligros de la ciudad de Trujillo. Convenio de Ejecución -Instituto de Investigación en Desastres y Medio Ambiente. 82-86,153- 168. pp.

Proyecta Consultoría. 2018a. Base de Sedimentos en la Cuenca Quebrada de San Idelfonso, Lima, Junio 2018. 11 -19 pp.

Proyecta Consultoría .2018b. Estudio Hidrológico de la Cuenca de San Idelfonso, Lima, Junio 2018. 14 -23 pp.

Medina, A. 2017. Peligros geológicos y geohidrológicos detonados por El Niño Costero 2017 en la Región La Libertad Cajamarca: Análisis geológico, geomorfológico y de peligros en la ciudad de Trujillo. Informe Técnico № A6791. INGEMMET. 28-42 pp.
Possia, N.; Cerne, M.; Campetella, C. 2015. Análisis preliminar de la tendencia de la presión en la Argentina. Centro de Investigación del Mar y de la Atmosfera-Facultad de Ciencias Exactas y Naturales -Buenos Aires Argentina. 1 pp.

Raffo, M. 1954. Variaciones de la napa freática en relación con la precipitación, la presión atmosférica y la temperatura. Buenos Aires -Argentina. 99-103 pp.

SENAMHI. 2014. El Fenómeno El Niño en el Perú. Ministerio del Ambiente-MINAM -Lima Perú. 9-17 pp.

SENAMHI. 2016. Popularización de la Meteorología en la educación Básica Regular Secundaria. Guía para el docente. CLIMANDES / COSUDE. Lima Perú. 91-94 pp

Zúñiga, I.; Crespo, E. 2016. Meteorología y Climatología. 2da. Edición. Editorial UNAD. Madrid. España. 68-78 pp. 